form deep gangrenous excavations between the bones of the forearm, in which muscular aponeuroses could be seen. She required a great deal of omnopon during the terminal stage of her illness to combat pain. On May 18, 1943, she died.

A post-mortem examination was unfortunately out of the question, and pathological confirmation is therefore lacking, but from the clinical course of the disease I think there is little doubt as to its true nature. Alternative diagnoses of rheumatic pericarditis, acute nephritis, septicaemia, and subacute bacterial endocarditis were all considered in turn as various signs arose, but none appeared entirely to satisfy the symptomatology:' The appearance of nodules and the extensive patches of gangrene on the arms, together with the obvious signs of a vascular lesion of the extremities, suggested the true diagnosis.

I am grateful to many for their help in this case, but particularly to Dr. W. O'Donnell, Prof. W. J. E. Jessop, and the late Prof. W. Boxwell, as well as to my house-physicians.

Dublin.

Cyril MurPhy, M.D., F.R.C.P.I.

\section{Tetanus following Appendicectomy}

The following case seems to be worth reporting because it developed after an operation and was successfully treated without opening up the wound.

The patient was a well-built man aged 29 , employed in the N.F.S. For two weeks before admission to hospital he had been complaining of pain and paraesthesia in the finger-tips of the right hand. A diagnosis of brachial neuritis had been made by a consultant physician.

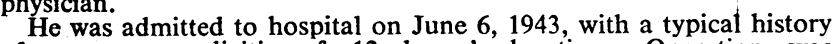
of acute appendicitis of 12 hours' duration. Operation was immediately performed, and an acutely inflamed appendix was revealed. The appendix was unobstructed and not perforated, and there was but little local peritonitis. A routine appendicectomy was performed without any unusual soiling of the peritoneum or abdominal wall with the contents of the appendix, the abdomen abdominal wall with the contents of the appendix, the abdomen
being closed without drainage. The operation was followed by an uneventful recovery until the ninth day, except for slight pain in the hand due to the brachial neuritis. On that day the patient complained of an aching pain in the lumbar region. Examination revealed tenderness of the lumbar muscles, but no spasm. Further questioning disclosed that the ache had been present in a mild form questioning disclosed that the ache had been present in
on the sixth day. A diagnosis of lumbago was made.

On the tenth day after operation there was slight stiffness of the jaw, and examination of the lumbar muscles showed a slight but definite spasm. A tentative diagnosis of tetanus was made, and this was substantiated later in the day by the development of difficulty in swallowing. The patient also complained of the sensation of in swallowing. The patient also complained of the sensation of to the onset of localized tetanic spasms. 180,000 i.u. of A.T.S. was immediately given intravenously, and chloral and potassium bromide, $20 \mathrm{gr}$. of each, were administered four-hourly.

The patient's condition deteriorated for the next two days, and he complained of tightness of the chest. Marked opisthotonos, abdominal rigidity, and trismus developed, but the bromide and chloral sufficed to keep the spasms local and infrequent. On the third day however, paraldehyde, $4 \mathrm{dr}$. twice a day per rectum, was required to control the spasms. Later the paraldehyde had to be given six-hourly in doses of $6 \mathrm{dr}$. to produce the required effect. Tribromethol was not used because of the increased liability to respiratory complications, and pethidine and chloretone were found to be less efficacious than paraldehyde.

The fifth day, when several generalized spasms were experienced, was probably the worst day. Then improvement began with disappearance of the rigidity of the abdominal wall. On the seventh day 100,000 i.u. of A.T.S. was given. As the condition improved lesssedative drugs were needed. Nutrition was maintained at a fairly adequate level throughout with glucose and milky fluids, without having to resort to a stomach tube. It was very important to feed the patient rapidly and efficiently just before the next dose of sedative was due. Then he was just conscious enough to feed. and yet the trismus was reduced and swallowing did not provoke the onset of generalized spasms.

A bacteriological diagnosis of tetanus was not made. There was a slight puqulent discharge from the wound, swabs from which revealed no evidence of tetanus bacilli. Their absence was probably due to the fact that a specimen could be obtained only from the superficial part of the wound. The wound was not explored.

There was no history of any injury, and the appendicectomy wound was the only possible focus for the growth of the tetanus bacilli. The catgut used at the operation was made by a reputable firm of manufacturers. When the tetanus became manifest it was not possible to trace the batch of catgut which was used and so test it for the presence of spores. Present-day catgut is an unlikely source of tetanus spores. It seems likely that the wound was contaminated by spores from the bowel lumen. It is stated by many authorities that tetanus spores are occasionally found in the lumen of the human bowel.

I wish to thank Mr. A. H. Southam for permission to publish this case and for his helpful criticism.

A. Jolleys, M.B., Сн.В.,

Surgical Registrar to Out-patients, Manchester Royal Infirmary.

\section{Reviews}

\section{MENTAL GROWTH OF CHILDREN}

The Mental Growth of Children from 2 to 14 Years. A Study of the Predictive Value of the Minnesota Preschool Scales. By Florence L. Minneapolis: University of Minnesota Press; London: Oxford University P.ess.

This is No. 19 of the series of monographs from the Institute of Child Welfare of the University of Minnesota. In it the results of repeated tests of the intelligence of the same children over a substantial period of time are described. The tests used are the Minnesota pre-school scales, revisions of the Stanford Binet and the Arthur performance scale, and the Minnesota College ability tests. At all ages the tests proved of predictive value. It was found that there is a good deal of inconstancy in the results of tests, especially in the early years, so that single tests are apt to be misleading and the longitudinal method here advocated would seem to be much more reliable. It proved difficult to follow many children through the whole 12-year period, but others of corresponding status were brought into the series, and the authors consider that their results are statistically valid.

Details of method and of results are given, and it would appear that individual mental growth does not always proceed at a fixed and uniform rate, though in the majority of cases constancy rather than inconstancy is the rule. Irregularity is most noticeable at early ages; there may be early precocity which does not last or early slowness with subsequent acceleration. Such irregularities are not necessarily due to external causes only. The old pre-school tests have not proved to be reliable, and new tests have been and still need to be worked out. The present study has shown that trustworthy indications of special abilities and differences of mental patterns can be detected at a much earlier age than was supposed. There is a suggestion that there may be a sex difference in the age at which mental tests begin to have a predictive value for future intellectual standing. It would appear that there is an initial feminine precocity which may be reversed later on. Further work is required and will be undertaken to amplify and confirm the findings.

This is a well-documented book with a useful bibliography at the end, and it will prove interesting and valuable to all who are concerned with the mental development of the child. The authors make it clear that there are many unsolved problems in the field of mental testing and that much work still remains to be done.

\section{THE HUMAN FOOT} The Foot. By Norman C. Lake, M.D., M.S., D.Sc., F.R.C.S. Third
edition. (Pp. 432 ; illustrated. 15s.) London: Baillière, Tindall and Cox. 1943 .

Mr. Lake's book has not undergone any drastic changes during his preparation of a third edition. The most significant changes are the closer attention given to those foot conditions which are seen more frequently in wartime, such as those associated with cold, damp, or excessive strains.

True frost-bite is stated to be a much rarer condition than trench foot, but with the present tendency to rapid movement in military tactics, compared with the more static methods in the last war, the incidence of trench foot is likely to fall. On the other hand, true frost-bite has become more common as the number of high-flying airmen increase. The reference to foot strain in industry and military service is important. Long hours of standing on a concrete floor bring many industrial workers to their doctors or to hospital, where this general excessive strain on a static foot is found to be a frequent cause of disability, though aggravated in many cases by more obvious deformities such as flat-foot. The soldier who complains of pain in the foot with no obvious cause may well respond to a more gradual introduction to "footslogging" than is customary.

Without doubt this is an exceedingly useful book for the doctor in general practice and the orthopaedic house-surgeon, and it should help the regimental medical officer to keep the Army soundly on its feet. 